\title{
Universiteit
}

Leiden

The Netherlands

\section{Diffusion in a surface: the atomic slide puzzle}

Saarloos, W. van; Frenken, J.W.M.; Gastel, R. van; Albada, S.B. van; Somfai, E.

\section{Citation}

Saarloos, W. van, Frenken, J. W. M., Gastel, R. van, Albada, S. B. van, \& Somfai, E. (2002). Diffusion in a surface: the atomic slide puzzle. Applied Physics A, 75, 11. Retrieved from https://hdl.handle.net/1887/5506

Version: $\quad$ Not Applicable (or Unknown)

License: $\quad$ Leiden University Non-exclusive license

Downloaded from: https://hdl.handle.net/1887/5506

Note: To cite this publication please use the final published version (if applicable). 


\title{
DIFFUSION ON AND IN SURFACES: THE ATOMIC SLIDE PUZZLE
}

\author{
J.W.M. FRENKEN, R. VAN GASTEL, E. SOMFAI*, W. VAN \\ SAARLOOS* \\ Kamerlingh Onnes Laboratory and "Instituut-Lorentz, Leiden \\ University, P.O. Box 9504, 2300 RA Leiden, The Netherlands
}

\section{Abstract}

This paper presents first results from scanning tunneling microscopy measurements, which show that a close-packed terrace of a metal surface can be far from static, even at temperatures as low as room temperature. We make the motion visible of the atoms in a $\mathrm{Cu}(001)$ terrace, by embedding a low density of In atoms in the first $\mathrm{Cu}$ layer. The peculiar characteristics of the motion of the In show that the diffusion of surface vacancies is responsible for a continual reshuffling of all the (In and $\mathrm{Cu}$ ) atoms in the first layer. A comparison with model calculations of the statistics of the vacancy-assisted motion of terrace atoms shows that there must be an attractive interaction between an embedded In atom and a vacancy, which makes the In atom somewhat more mobile than a $\mathrm{Cu}$ surface atom. Such an attraction is indeed found in Embedded Atom calculations.

\section{Introduction}

Usually, surface mobility is thought to be restricted completely to the steps and kinks on crystal surfaces. These sites form the natural locations for atoms to 'come and go'. The resulting step motion can be observed both on the atomic and on mesoscopic length scales with various types of, such as e.g. scanning tunneling microscopy microscopy (Jeong et al., 1999; Zhang et al., 1997; Kuipers et al., 1993). Dynamic step rearrangements can also be responsible for the two-dimensional diffusive motion of adatom and vacancy islands (Morgenstern et al., 1995). By contrast, those atoms in the close-packed terraces that are not in the direct vicinity of steps are often considered to be completely 
static, since they are tightly packed by their neighbors. Although suggestions have been made that also surface vacancies might be responsible for selfdiffusion of islands (Hannon et al., 1997), there are no experimental techniques to date, which can follow the diffusion of naturally occurring vacancies in a close-packed metal surface. Available forms of microscopy and spectroscopy either suffer from a lack of spatial resolution, or a lack of temporal resolution, or both.

Here we show that is possible to use scanning tunneling microscopy to see the effect of diffusing vacancies in a metal surface indirectly, via the motion of impurity atoms. For this purpose, the impurities should have the special property that they embed themselves substitutionally in the outermost crystal layer. One of the surface-impurity combinations, which is known to behave like this, is that of $\mathrm{In}$ on $\mathrm{Cu}(001)$ (Breeman et al., 1992,1993). At temperatures just below room temperature In atoms deposited on $\mathrm{Cu}(001)$ are incorporated in substitutional terrace sites. We observe a remarkable mobility of the embedded In atoms. From the statistics of the motion, we conclude that the In atoms are assisted by another 'particle' in their surface diffusion, and we show that the most likely candidate for this assisting particle is a vacancy in the $\mathrm{Cu}(001)$ surface. From our observations of the motion of In atoms we conclude that also the $\mathrm{Cu}$ terrace atoms have a surprisingly high mobility, even at room temperature.

In this paper we present preliminary results of this work. A more complete account will be given in a forthcoming publication.

\section{Experimental}

The experiments were performed with a variable temperature scanning tunneling microscope (STM) (Hoogeman et al., 1998), in ultrahigh vacuum (UHV). A Cu single crystal of $99.999 \%$ purity was mechanically polished parallel to the (001)-plane, and heated in an $\mathrm{Ar} / \mathrm{H}_{2}$ atmosphere to remove sulfur traces from the bulk. The $\mathrm{Cu}$ surface was further cleaned in UHV by several tens of cycles of sputtering with $600 \mathrm{eV} \mathrm{Ar}^{+}$ions and annealing to $675 \mathrm{~K}$. The surface was regularly exposed to a few Langmuir of $\mathrm{O}_{2}$ to remove carbon from the surface. STM images showed a well-ordered surface with terrace widths up to $8000 \AA$. In was deposited in situ from a Knudsen cell either at cryogenic temperatures, or at room temperature. In this paper we will restrict ourselves to those cases where In was deposited at room temperature. 


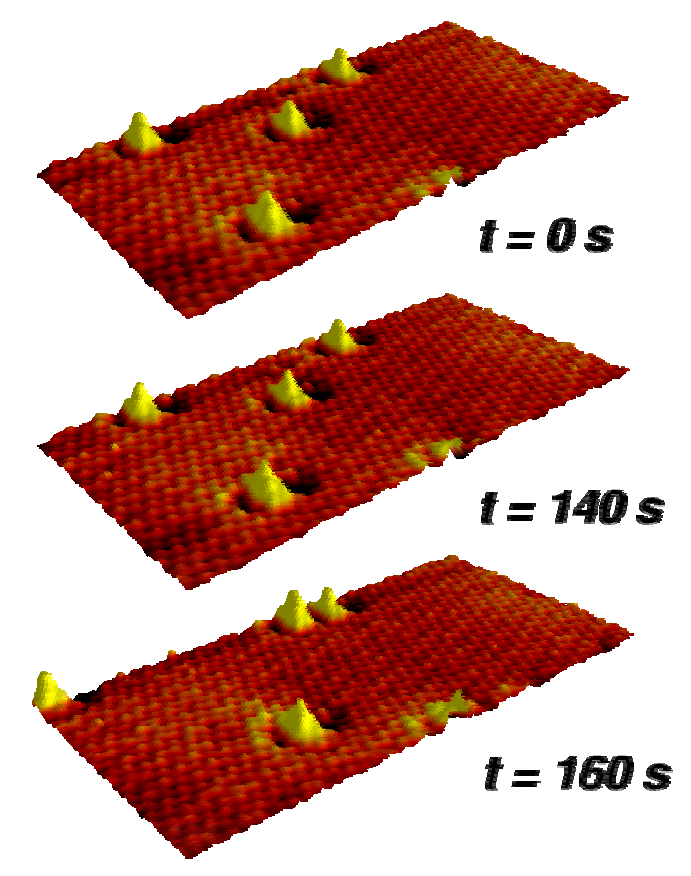

Figure 1. Three STM-images of $52 \times 103 \AA^{2}$ selected from a movie measured at room temperature, illustrating the unusual diffusion of embedded indium atoms. The fine corrugation is due to the $\mathrm{Cu}(001)$ lattice. The four larger protrusions are embedded In atoms. In the time interval of $140 \mathrm{~s}$ between images (a) and (b), the four imaged In atoms have not moved. During the next $20 \mathrm{~s}$, all four In atoms have been displaced over several lattice spacings (c). $\left(\mathrm{I}_{\mathrm{t}}=0.9 \mathrm{nA}, \mathrm{V}_{\mathrm{t}}=0.58 \mathrm{~V}\right)$

\section{Observations}

The STM images obtained at room temperature after deposition of In show that the In atoms are introduced into the outermost $\mathrm{Cu}$ layer via the steps on the $\mathrm{Cu}(001)$ surface. We have observed that the embedded In atoms slowly diffuse away from the steps, to homogeneously populate the terraces only after several hours. The observed diffusion distances are orders of magnitude larger than the root-mean-square step fluctuations occurring on the same time scale at room temperature, which implies that the In is not simply incorporated in the first $\mathrm{Cu}$ layer as a consequence of the step fluctuations of the $\mathrm{Cu}$ surface. 

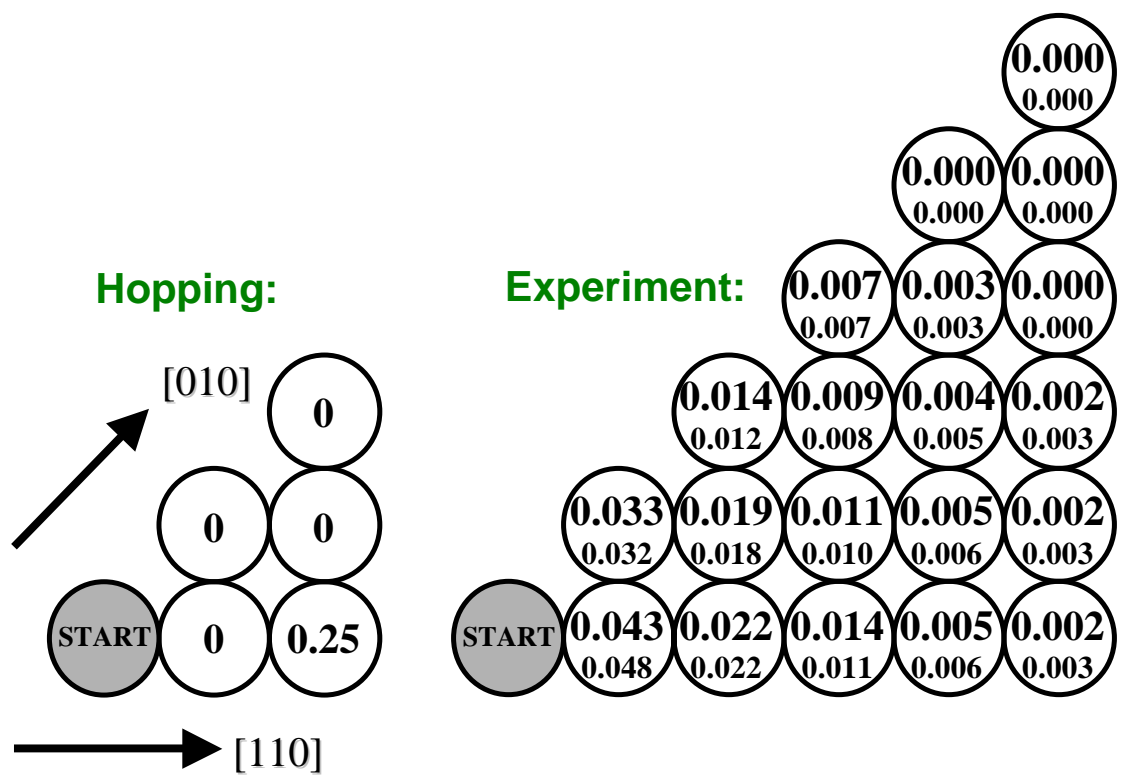

Figure 2. Jump vector distributions. The right panel shows the distribution for embedded $\mathrm{In}$ atoms in $\mathrm{Cu}(001)$. The numbers correspond to the probabilities for jumps of an In atom from its starting position to each of the indicated non-equivalent lattice sites. The upper number on each site is the probability measured from STM-movies at $320 \mathrm{~K}$. The lower number is the result of the model calculation described in the text. For comparison, the jump vector distribution expected for the case of simple hopping is shown in the left diagram.

Figure 1 shows three images taken from an STM movie* at $320 \mathrm{~K}$, after deposition of $3 \%$ of a monolayer of In on the clean $\mathrm{Cu}(001)$ surface. The smallscale corrugation corresponds to the atomically resolved $\mathrm{Cu}$ surface, while the larger protrusions are individual embedded In atoms. The vertical scale is exaggerated; the In atoms stick out only $0.4 \AA$ above the surface. What is illustrated by Figure 1, and what is observed in all our movies of the motion of In embedded in $\mathrm{Cu}(001)$, both at $320 \mathrm{~K}$ and at other temperatures, is that the In atoms move via long jumps of more than a single lattice spacing,

\footnotetext{
* The full movie can be viewed on http://www-lion.leidenuniv.nl/sections/cm/ groups/interface
} 
separated by long time intervals. In addition, the movies show that there is a strong tendency for nearby In atoms to jump at the same time.

From the STM movies we have measured the distribution of jump lengths of the embedded indium atoms, which is displayed in the diagram of Figure 2. The diagram shows the triangle of jump orientations between the [010] and [110] azimuths; the upper number shown for each lattice point corresponds to the average probability measured for the jump vector corresponding to that lattice position and the probabilities for the 3 or 7 equivalent jump vectors in the symmetry related directions that are not shown in the diagram. It is clear that the distribution extends well beyond a single lattice spacing, and that there is even a significant probability for the indium atoms to make jumps as long as five lattice spacings.

\section{Interpretation}

The long jumps and the high probability of nearby indium atoms to jump simultaneously are hallmarks of a diffusion mechanism in which the indium is assisted by another particle, which diffuses so rapidly that it remains invisible to the STM. The simplest scenario is that in which the In moves over a single lattice spacing by changing places with the assisting particle. Even if the assisting particles perform completely random walks, the In has a high probability to move over several lattice spacings as the result of the passage of a single assisting particle, simply because the random walk has a high probability to bring the particle back to the In atom after every encounter (see below). The fact that a single vacancy will usually encounter various In atoms, naturally explains the tendency for nearby In atoms to jump at the same time.

$\mathrm{Cu}$ adatoms and (surface) vacancies can both be considered as natural candidates for the assisting particle. Figure 3 shows how an encounter of an embedded In atom with a $\mathrm{Cu}$ adatom as well as an encounter with a surface vacancy can lead to a displacement of the In atom over a single atomic spacing. On the basis of the displacement statistics of the In atoms, we cannot distinguish between the two scenarios. However, we can decide between the two possibilities by observing the incorporation pathway of In upon deposition on the $\mathrm{Cu}(001)$ substrate. As mentioned above, we observe that freshly deposited In atoms cannot invade the $\mathrm{Cu}(001)$ terrace directly, but they only reach substitutional terrace sites via the steps. This immediately rules out the possibility of adatom-assisted motion, since the exchange of an In adatom with a terrace $\mathrm{Cu}$ atom is a necessary ingredient of such motion (cf. Figure 3).

The mechanism that we end up with is illustrated in Figure 4. The twodimensional random walk of a single surface vacancy leads to the multiple 

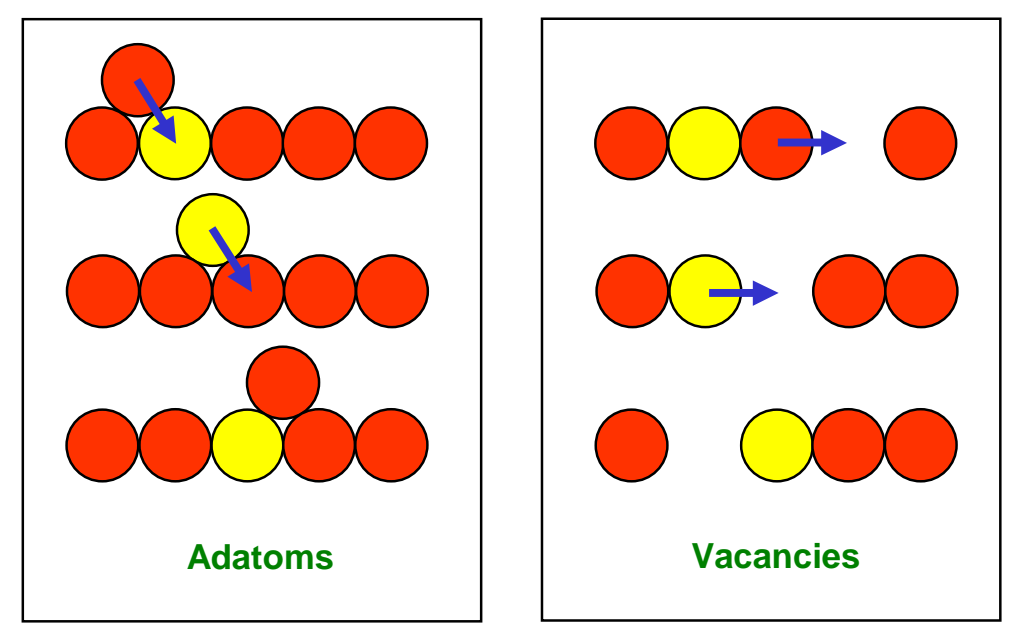

Figure 3. Two scenarios for assisted diffusion of embedded In atoms. In the scenario on the left, the In atom moves by an exchange process with a $\mathrm{Cu}$ adatom. After the first step, the In atom has temporarily become an adatom itself. A second exchange step embeds the In atom again into the first $\mathrm{Cu}$ layer. In the process the In atom is moved over one atomic spacing. In the scenario on the right, the In atom moves as the result of the passage of a surface vacancy.

displacement of an embedded In atom. Note that also many of the terrace $\mathrm{Cu}$ atoms undergo similar, large displacements. In the proposed mechanism, the length of the long jumps of the indium atoms depends on the average number of times that a single vacancy changes places with an indium atom, and we associate the frequency of the (long) indium jumps with the frequency with which the indium is encountered by new vacancies.

\section{Model}

The simplest model description of the vacancy-assisted motion treats the In atom as a tagged $\mathrm{Cu}$ atom. The probability of a vacancy to make single 'jumps' in each of the four directions is simply $25 \%$. Based on these probabilities, it is easy to calculate the four probabilities for a vacancy, after an initial encounter with an In atom, to return to the In atom from each of the four relevant directions: from the same side $P_{1}$, from the opposite side $P_{2}$ or from one of the two sideways directions $P_{3}=P_{4}$. If the $\mathrm{Cu}(001)$ terrace is of infinite size, these 


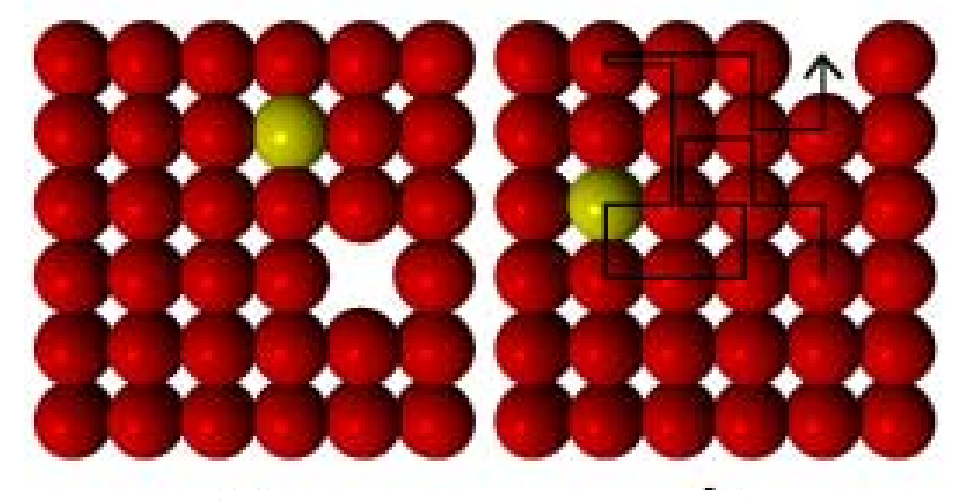

Figure 4. Ball model illustrating the vacancy-mediated diffusion mechanism of the In atoms. The random walk of a single vacancy results in the multiple displacement of the In atom.

four return probabilities add up to unity: the vacancy will return to the In atom infinitely many times and will therefore cause a diverging displacement of the In atom. However, on a real surface, the vacancy will have a finite lifetime, so that also the displacement of the In atom resulting from the passage of a single vacancy will be finite. The vacancy most probably originates from a step and it will most probably also terminate its 'life' at a step. In our toy model, the finite lifetime of the vacancy is introduced in the form of a fifth probability $P_{a n n}$, which is the probability of the vacancy to be annihilated before it returns once more to the In atom. We treat this probability as a free parameter in an attempt to fit the experimentally determined jump vector distribution. In Figure 2 we compare the theoretical and experimental distributions. The model provides an excellent fit for a value of the annihilation probability of $P_{a n n}=0.027$. The shape of both the measured and the calculated distributions in Figure 2 is significantly different from the two-dimensional Gaussian distribution, expected in the case of ordinary, i.e. non-assisted diffusion. In fact, the shape is very close to that of a modified Bessel function of order zero. As we will demonstrate in a future publication, it is easy to show that this shape is indeed characteristic of the type of assisted diffusion considered here, where the assisting particle has a limited lifetime.

The value expected for $P_{a n n}$ for the average step separation observed in the experiment of approximately 400 lattice spacings is 0.23 . This is much higher than the value obtained from the fitting described above. The dependence of $P_{a n n}$ on the average terrace width is extremely weak (a logarithmic dependence 
has been derived in a somewhat different context by Brummelhuis et al., 1988). In order to reach the low value of 0.027 , found above, the average terrace width would have to be as large as $10^{25}$ atomic spacings, which is completely unphysical! This result illustrates an important flaw of the simple model, which is the complete neglect of the chemical difference between In and $\mathrm{Cu}$. $\mathrm{An}$ increase by an order of magnitude in the average number of returns of the vacancy to the In atom is easily obtained as a consequence of the modifications of the diffusion barriers of the vacancy in the vicinity of the In atom, with respect to the normal barrier for vacancy diffusion in the clean $\mathrm{Cu}(001)$ terrace. Indeed, such changes in activation barrier are found in calculations using the Embedded Atom Model (we have used the potential described by Breeman et al., 1992, 1993), which show a noticeable attractive interaction between the vacancy and the (large) In atom. When we modify the model to include the EAM diffusion barriers of the vacancy in the vicinity of the In atom, and if we fix the lifetime of the vacancy via the constraint that the vacancy's random walk terminates once it reaches the nearest step, for a step separation of 400 lattice spacings, we obtain a distribution that is almost identical to the equal-barrier result shown in Figure 2, i.e. with an equally good match to the measured distribution of jump lengths, but this time without any adjustable parameters in the model. Full details of this calculation will be given in a forthcoming publication.

\section{Conclusion}

In summary, STM measurements show that In atoms, embedded in the $\mathrm{Cu}(001)$ surface, perform two-dimensional diffusive motion that cannot be explained by step fluctuations. The measurements strongly suggest that the motion is caused by the two-dimensional diffusion of surface vacancies in the $\mathrm{Cu}$ surface. These observations imply that not only the In atoms exhibit a high mobility in the surface, but that also all $\mathrm{Cu}$ terrace atoms are continually 'on the move'. We expect that in this respect $\mathrm{Cu}(001)$ is not very different from other close-packed surfaces of $\mathrm{Cu}$ and from other materials, which means that we have to regard all close-packed metal terraces as quite dynamic, even at temperatures as low as room temperature. 


\section{Acknowledgment}

This project is part of the research program of the "Stichting voor Fundamenteel Onderzoek der Materie (FOM)", which is financially supported by the "Nederlandse Organisatie voor Wetenschappelijk Onderzoek (NWO)".

\section{References}

Breeman, M., and Boerma, D.O. (1992), "Sites, mobilities and cluster formation of In atoms on a stepped $\mathrm{Cu}(100)$ surface", Phys.Rev. B 46, 1703-1709.

Breeman, M., and Boerma, D.O. (1993), "Atomic mobilities on a stepped $\mathrm{Cu}(100)$ surface”, Surf.Sci. 287/288, 881-885.

Brummelhuis, M.J.A.M., and Hilhorst, H.J. (1988), "Single vacancy induced motion of a tracer particle in a two-dimensional lattice gas", J.Stat.Phys. 53, 249-278.

Hannon, J.B., Klünker, C., Giesen, M., Ibach, H., Bartelt, N.C., and Hamilton, J.C. (1997), "Surface self-diffusion by vacancy motion: Island ripening on $\mathrm{Cu}(001)$ ", Phys.Rev.Lett. 79, 2506-2509.

Hoogeman, M.S., Glastra van Loon, D.,Loos, R.W.M., Ficke,H.G., De Haas, E., Van der Linden, J.J, Zeijlemaker, H., Kuipers, L., Chang, M.F., Klik, M.A.J., and Frenken, J.W.M. (1998), "Design and performance of a programmabletemperature scanning tunneling microscope", Rev.Sci.Instr 69, 2072-2080.

Jeong, H.-C. and Williams, E.D. (1999), "Steps on surfaces: Experiments and theory", Surf.Sci.Rep. 34, 171-294.

Kuipers, L., Hoogeman, M.S., and Frenken, J.W.M. (1993), "Step dynamics on $\mathrm{Au}(110)$ studied with a high-temperature, high-speed scanning tunneling microscope", Phys.Rev.Lett. 71, 3517-3520.

Morgenstern, K., Rosenfeld, G., Poelsema, B., and Comsa, G. (1995), "Brownian motion of vacancy islands on $\operatorname{Ag}(111)$ ", Phys.Rev.Lett. 74, 20582061.

Zhang, Z., and Lagally, M.G. (1997), "Atomistic processes in the early stages of thin-film growth”, Science 276, 377-383. 